\title{
Excitons bound by photon exchange
}

\author{
Erika Cortese, ${ }^{1,5}$ Ngoc Linh Tran, ${ }^{2,5}$ Jean-Michel Manceau, ${ }^{2}$ Adel Bousseksou, ${ }^{2}$ Iacopo \\ Carusotto, ${ }^{3}$ Giorgio Biasiol, ${ }^{4}$ Raffaele Colombelli, ${ }^{2,}{ }^{*}$ and Simone De Liberato ${ }^{1, \dagger}$ \\ ${ }^{1}$ School of Physics and Astronomy, University of Southampton, Southampton, SO17 1BJ, United Kingdom \\ ${ }^{2}$ Centre de Nanosciences et de Nanotechnologies (C2N), CNRS UMR 9001, \\ Univ. Paris-Sud, Université Paris-Saclay, 91120 Palaiseau, France \\ ${ }^{3}$ INO-CNR BEC Center and Dipartimento di Fisica, Universita di Trento, I-38123 Povo, Italy \\ ${ }^{4}$ Laboratorio TASC, CNR-IOM, Area Science Park, \\ S.S. $14 \mathrm{~km}$ 163.5, Basovizza, I-34149 Trieste, Italy \\ ${ }^{5}$ These authors contributed equally to this work
}

In contrast to interband excitons in undoped quantum wells, doped quantum wells do not display sharp resonances due to excitonic bound states. In these systems the effective Coulomb interaction between electrons and holes typically only leads to a depolarization shift of the singleelectron intersubband transitions [1]. Nonperturbative light-matter interaction in solidstate devices has been investigated as a pathway to tune optoelectronic properties of materials $[2,3]$. A recent theoretical work [4] predicted that, when the doped quantum wells are embedded in a photonic cavity, emission-reabsorption processes of cavity photons can generate an effective attractive interaction which binds electrons and holes together, leading to the creation of an intraband bound exciton. Here we spectroscopically observe such a bound state as a discrete resonance which appears below the ionisation threshold only when the coupling between light and matter is increased above a critical value. Our result demonstrates that two charged particles can be bound by the exchange of transverse photons. Light-matter coupling can thus be used as a novel tool in quantum material engineering, tuning electronic properties of semiconductor heterostructures beyond those permitted by mere crystal structures, with direct applications to mid-infrared optoelectronics.

Improvements in resonator design and fabrication have allowed to investigate the strong light-matter coupling regime in a multitude of solid-state devices [6]. In such a regime the excitations of the system are not photons or matter resonances, but polaritons, hybrid quasiparticles which are linear superpositions of them.

If the light-matter coupling is large enough to couple multiple matter resonances, characterised by different spatial wavefunctions to the same photonic mode, the ensuing polariton wavefunction will be a linear superposition of the uncoupled ones, possessing its own unique features, a priori different from those of the uncoupled modes. Such a phenomenon, referred to as very strong coupling [7-9] has only recently been experimentally
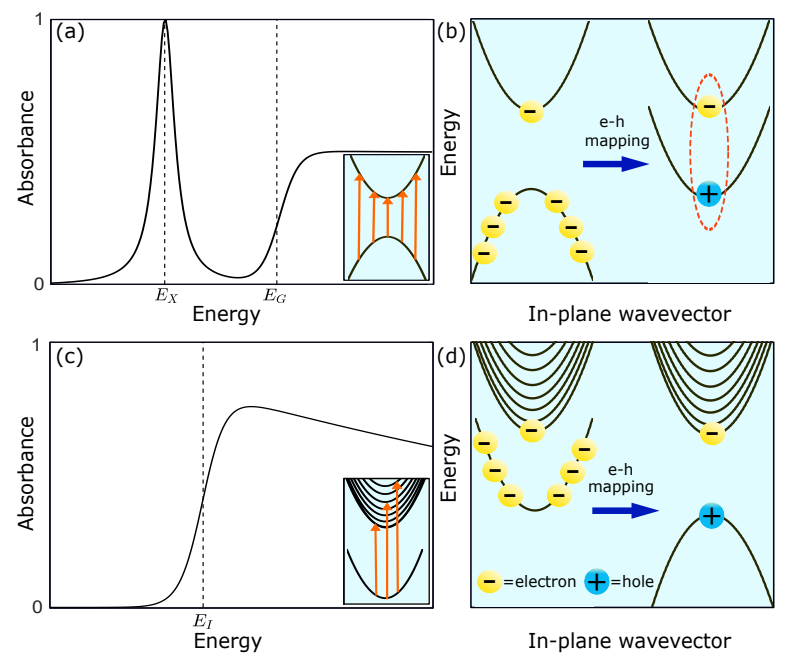

Figure 1. Coulomb effect in doped and undoped quantum wells. In panel (a) we sketch the interband absorbance of an undoped semiconductor QW, dominated by an excitonic resonance $\left(E_{X}\right)$ below the bandgap energy $\left(E_{G}\right)$ and the electron-hole continuum above it. In the inset we schematically illustrate the origin of the continuum part of the spectrum, understandable in a single-electron picture as interband transitions of electrons with different in-plane wavevectors. In panel (b) we illustrate the standard electron-hole mapping, allowing us to describe a single electron vacancy in the valence band as a hole with positive charge and mass. We can thus understand the excitonic peak as an hydrogenoid bound state of an electron and a positively charged hole, schematised by the red dashed line enclosing the electron-hole pair. In panel (c) we sketch instead the intersubband absorbance of a doped QW containing only one localized state, and a continuum of states above the first ionization energy of the QW $\left(E_{I}\right)$. Only the bound-to-continuum absorbtion is present, and no excitonic peak is visible [5]. As shown in the inset the continuum is, in this case, due to each electron having multiple possible delocalised final states. In panel $(d)$ we show how the initially filled electron subband has in this case a positive effective mass and the electron-hole mapping leads to a positively charged hole with negative effective mass, unable to form a bound state with the electron under the sole effect of the two-body Coulomb interaction.

demonstrated in inorganic microcavities, where it can be 
observed as a change of the exciton radius $[10,11]$.

The question on whether such a mechanism can be pushed to the extreme, non-perturbatively modifying the excitation wavefunction and creating localised bound states from delocalised ones, has recently been theoretically investigated, leading to the prediction of discrete resonances appearing below the continuum ionization threshold for large enough values of the light-matter coupling strengths [4]. The lower edge of the continuum corresponds to free electrons with no kinetic energy. The discrete resonances below such energy generated by the coupling with the photonic resonator have thus to be bound, as electrons have not enough energy to escape to infinity.

The system investigated in Ref. [4], a set of microcavity embedded $n$-doped semiconductor quantum wells (QW), is particularly suitable to highlight this physics. First, it allows to achieve large light-matter coupling strengths $[12,13]$, which can be tuned by changing the electronic density in the QW [14]. Second, it is possible to design QWs narrow enough to have a single localised electronic subband, and as such no bound-to-bound intersubband transitions. Third, in such a system the Coulomb interaction does not create bound states. The two last points imply that the bare QW does not present any discrete resonance at all, only a continuum absorption band at frequencies larger than the ionization threshold. Initially demonstrated in Ref. [1], the absence of Coulombbound intersubband states stems from the quasi-parallel dispersion of the two electronic subbands, which leads to a repulsive electron-hole interaction.

This is in stark contrast to the case of interband transitions at shorter wavelengths, where the electron-hole interaction is attractive and leads to the creation of narrow resonances out of the electron-hole continuum already in the absence of polaritonic effects: excitons in undoped samples [15] and Mahan singularities in doped ones [16]. Polariton formation can thus modify the existing resonances as in Ref. [11], but it doesn't lead to the creation of novel localised electronic resonances.

In Fig. 1 we present a sketch of this phenomenon, useful to understand where the analogy with the most widely studied case of interband transitions in undoped QWs breaks down. In the case of interband transitions in undoped QWs the electrons participating in the transition initially occupy the valence band, which has a negative effective mass. In the case of intersubband transitions in doped QWs instead, the same role is played by the first, partially-filled conduction subband, which has instead a positive effective mass. Under the usual electron-hole mapping [17] this leads to a positively charged hole with a negative effective mass. In GaAs QWs the effective electron mass in the excited subband $m_{2}^{*}$ is larger than the mass in the first subband $m_{1}^{*}[1]$. This leads to a negative reduced mass of the intersubband electron-hole pair $m_{r}^{-1}=m_{2}^{*-1}-m_{1}^{*-1}$. In the presence of any attractive

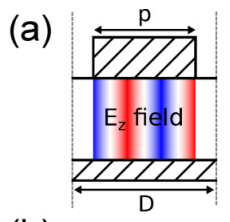

(b)
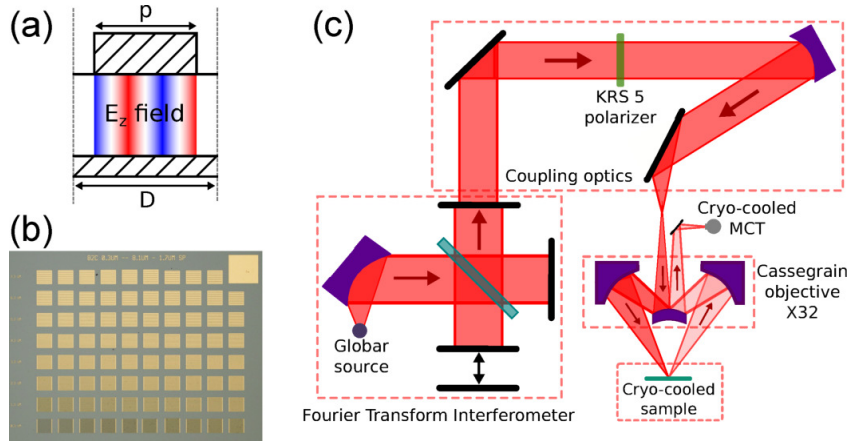

Figure 2. Schematics of the experimental setup. (a) Distribution of the electric field component orthogonal to the metallic layers for one period of the structure and for the mode $\mathrm{TM}_{02}$ of the ribbon resonator. (b) Optical microscope image of a typical sample set. Each device features a different finger width $p$. The top right device is a fully metallized surface that serves as mirror reference. (c) Scheme of the experimental setup employed for the reflectivity measurements. It consists of a mid-IR microscope connected to an FTIR spectrometer. The source is a thermal globar lamp, and the detector is a liquid nitrogen cooled MCT. The incident beam is polarized normally to the metallic fingers, and the system operates in reflection. A compact, thin cryostat permits to perform the measurements down to a temperature of $78 \mathrm{~K}$.

two-body potential, a negative reduced mass results in a repulsive electron-hole interaction, which in turn cannot create bound states. This can be intuitively understood observing that the classical equation of motion for the electron-hole pair is symmetric under sign exchange of both hole charge and reduced mass.

Note that this argument considers only the twobody interactions between electrons and holes. Interactions mediated by bosonic fields instead can and do create attractive interactions between particles of the same charge. This is the case for the light-matter interaction considered here, and most famously demonstrated in a many-body context by the phenomenon of Cooper pairing.

In order to provide evidence of photon-mediated bound states we realised a system similar to the one described in Ref. [4], composed of 13 GaAs/AlGaAs quantum wells embedded in highly-confining, grating-shaped gold microcavity resonators. More details on the sample can be found in the Methods. The resonators are 1D ribbons (or 1D patch cavities [18]), and the electromagnetic field, as sketched in Fig. 2(a), is almost completely confined below the metallic fingers. The QWs are chosen thin enough to have a single trapped conduction subband, as the presence of a second one would lead to the creation of intersubband polaritons [19]. Not only this would complicate the analysis of the data, but the presence of a bound-to-bound transition would almost saturate the available oscillator strength, leaving little for the 
bound-to-continuum transition we aim to measure. For the purpose of checking this important point, we fabricated two samples, HM4229 and HM4230, differing in QW width and doping. Sample HM4229 contains 4-nmthick GaAs QWs $\left(L_{\mathrm{QW}}=4 \mathrm{~nm}\right)$, each doped at a density $5 \times 10^{12} \mathrm{~cm}^{-2}$, while sample HM4230 contains $3.5-\mathrm{nm}$ thick QWs $\left(L_{\mathrm{QW}}=3.5 \mathrm{~nm}\right)$ doped at $4.77 \times 10^{12} \mathrm{~cm}^{-2}$.

As it is shown in the inset of Fig. 3, bound-to-bound and bound-to-continuum transitions between different single-particles states of the quantum well potential undergo opposite frequency shifts when the quantum well width is reduced: the former ones blue-shift, while the latter ones red-shift. This allows us to assess the nature of the optical transition by looking at the transmission spectra of the two samples before the gold deposition, shown in the main panel of Fig. 3.

Here we observe a very broad absorption that - being transverse magnetic polarized - stems from the doped QWs. We also observe some narrower feature around $140 \mathrm{meV}$ which can be identified as the edge of the continuum. Crucially, this feature does not blue-shift with decreasing QW width. It shows a transfer of spectral weight toward the red, while a bound-to-bound transition would have led to a blue-shift of the order of tens of meVs, proving the bound-to-continuum nature of the transitions in the bare QWs.

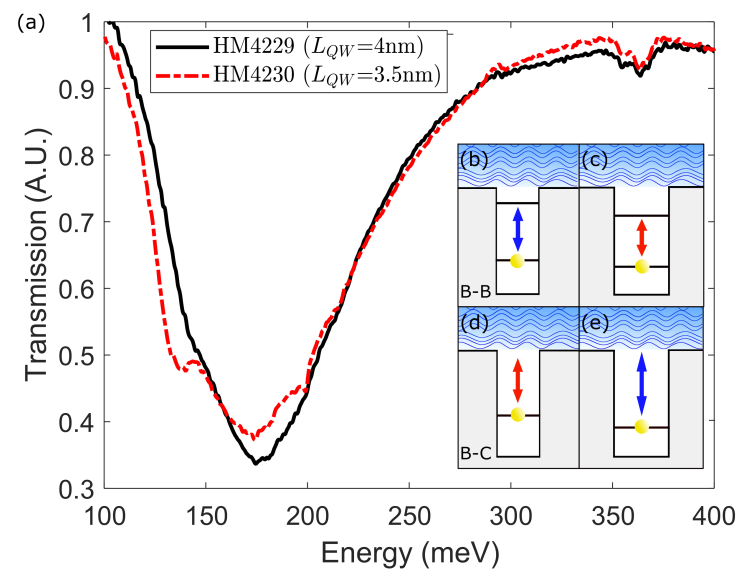

Figure 3. Bound-to-continuum nature of the optical transition in an isolated quantum well with no surrounding cavity. (a) The black solid line is the transmission measured at $300 \mathrm{~K}$ from the sample HM4299, with $L_{\mathrm{QW}}=4$ $\mathrm{nm}$. The red dashed line represent instead sample HM4230, with $L_{\mathrm{QW}}=3.5 \mathrm{~nm}$. In the inset we sketch how the transition frequency shifts as a function of the quantum well width for bound-to-bound (b,c) and bound-to-continuum (d,e) transitions. Right panels (c,e) differ from the left ones $(b, d)$ for a thicker quantum well along the growth axis. The black horizontal lines indicate the single-particle bound states, while the wiggly blue lines indicate the delocalized single-particle states above the ionization threshold.
Each sample was fabricated into metal-semiconductormetal grating resonators with period $D$ and width of the metallic finger $p$, as sketched in Figs. 2(a) and (b), and following a procedure similar to the one in Ref. [20]. As the electromagnetic field is extremely localized below the metallic fingers, the system essentially behaves as a Fabry-Perot cavity of length $p$ [18]. More details can be found in the Methods.

We fabricated several grating-based devices, $200 \times 200$ $\mu \mathrm{m}^{2}$ large, with $p$ ranging from $800 \mathrm{~nm}$ to $5 \mu \mathrm{m}$, in order to span a vast range of frequencies with the resonant cavity modes (see Fig. 2(b)). Each device was then measured in reflectivity, at a temperature of $78 \mathrm{~K}$, using a Fourier-transform infrared (FTIR) microscope equipped with a very compact cryostat. A scheme of the experimental setup is shown in Fig. 2(c). The main result of this work is reported in Fig. 4, where in panel (a) we plot the $78 \mathrm{~K}$ reflectivity map of the HM4229 sample as a function of the bare resonator frequency. Details on the spectroscopic characterization and the data analysis are provided in the Methods. While above the ionization threshold, that is materialized by a black horizontal dashed line, we can observe an absorption continuum, below it a narrow polaritonic resonance appears. It is red-shifted by more than $20 \mathrm{meV}$ with respect to the bare resonator. Overlaid on the colormap we plot the peak frequencies obtained by a multiple Lorentzian fit of the data. Red triangles and blue squares are used respectively for frequencies below and above the identified ionization threshold marked by black dashed lines. For comparison, the green circles mark the frequency of the bare resonator, measured on an undoped sample (data in Supplementary Information). In panel c) the respective linewidths are shown as a function of the resonant mode frequency.

Below the ionization threshold it appears that the lifetime of the discrete polaritonic mode is mainly limited by the resonator lifetime. Above it, instead, we have a bound-to-continuum spectrum in which only very broadened and undefined features can be identified. To better illustrate this finding, in Fig. 4(b) we plot the reflectivity spectra of the doped sample (red curves) for different values of the bare resonator frequency, together with the resonator resonance measured on a undoped sample (green curves). From such a figure we can clearly observe how the doped sample develops a discrete resonance below the continuum edge, while an identical, but electromagnetically uncoupled sample presents none.

Note that in Fig. 4(a) both the narrow discrete mode below the continuum edge (red) and the extracted peak of the broad continuum above it (blue) are at frequencies lower than the bare resonator (green). Moreover, they have very different linewidths which remain well separated for all cavity energies. These features are not compatible with a standard (bound-to-bound) polaritonic description, in which due to mode repulsion 

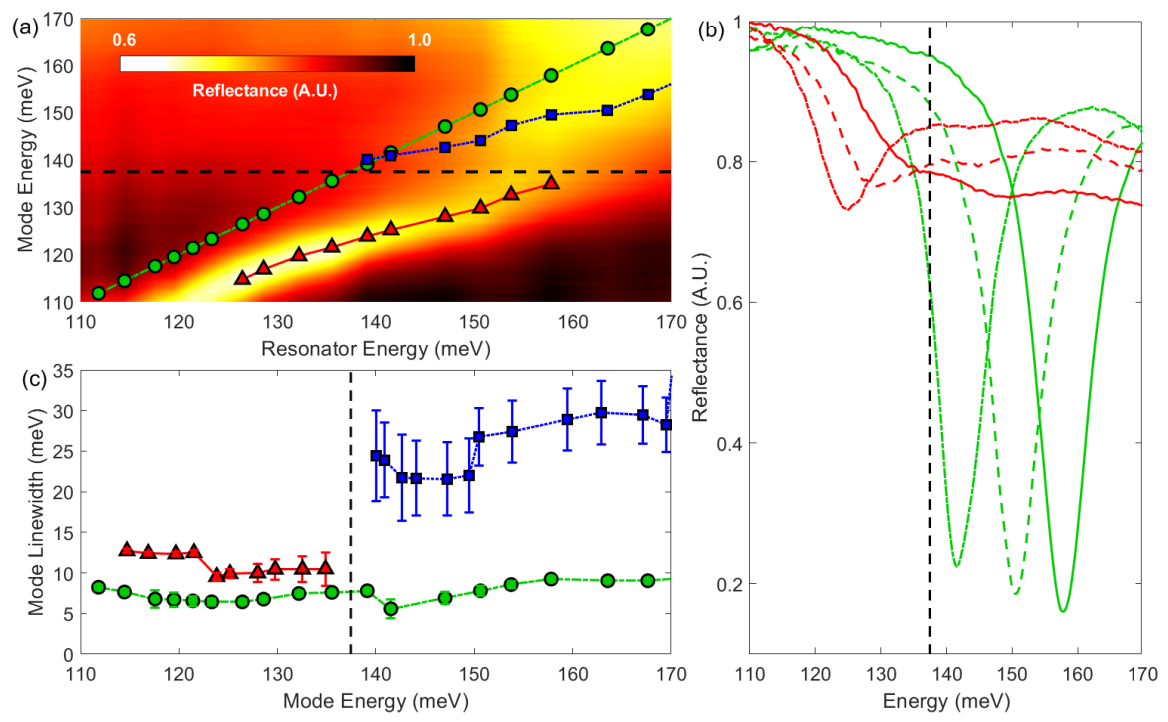

Figure 4. Experimental reflectivity data (a) Reflectance data from the doped sample HM4229 as a function of the resonator frequency. Superimposed we show the peak position extracted from the data, using blue squares and red triangles for the datapoints respectively below and above the edge of the continuum. With green dot we mark instead data from the undoped sample taken as reference for the cavity energy. (b) Reflectance data for doped sample HM4229 (red) and the empty cavity (green) for resonator frequencies $\hbar \omega_{c}=157.8 \mathrm{meV}$ (solid), $\hbar \omega_{c}=147 \mathrm{meV}$ (dashed), and $\hbar \omega_{c}=141.5 \mathrm{meV}$ (dot dashed). (c) Linewidths for the different modes as a function of the mode energy. Error bars are 95\% confidence intervals, determined via a multiple Lorentzian peak fitting procedure. In all the panels the dashed black line marks the first ionization energy of the QW, marked as $E_{I}$ in Fig. 1(d).

both the light and matter resonances are between the polaritonic modes. Moreover in a simple Hopfield model [21] the coupled modes linewidth are weighted averages of the bare light and matter ones and they should thus cross across the anticrossing. Data on sample HM4230 in strong coupling are reported in Supplementary Information. They are similar to the data presented here on HM4229 and point at the same conclusions.

Using the theory of Ref. [4] such a hybrid discrete state can be written as a polariton whose electron density relative to the ground state one is given by

$$
\Delta N(z)=P\left[\left|\psi^{e}(z)\right|^{2}-\left|\psi^{g}(z)\right|^{2}\right],
$$

where $P$, varying between 0 and 1 , is the weight of the polaritonic matter component, $\psi^{g}(z)$ is the normalised wavefunction of an electron in its ground state, and $\psi^{e}(z)$ is the wavefunction of a localised electronic state generated by the light-matter interaction. In Fig. 5(a) we use the model of Ref. [4] to reproduce the observed reflectivity spectrum and compare it to the experimental data. Using the parameters of such a simulation we calculated the matter weight $P$, which we plot in Fig. 5(b). From such a figure we can see that the discrete resonance below the ionization threshold is clearly defined for nonvanishing values of $P$, demonstrating a substantial occupation of the light-generated electronic wavefunction $\psi^{e}(z)$.
With this experiment we demonstrated the possibility to strongly couple a ionizing transition to a photonic resonator. As theoretically predicted, this results in a non-perturbative modification of the system's electronic structure. This leads to the appearance of a hybrid polaritonic excitation whose matter part is a bound state generated by the light-matter interaction, consisting of an electron and an hole kept together by their interaction with the transverse electromagnetic field. The experiment reported in this work measures only the optical properties of the system. However, an immediate question arises: can these bound states conduct electrical currents? A positive answer would imply they can act as discrete and tunable current channels into bright states, and as such it has been predicted they could allow for dramatic increase in the efficiency of intersubband midinfrared emitters [22]. Initial work in this direction, albeit for a bound-to-bound transition, has been recently reported in mid-infrared detectors operating in the strong light-matter coupling regime [23].

The possibility of tuning material properties through coupling to a microcavity photonic field has been much discussed lately, with particular attention for chemical and structural properties [24-26]. Beyond the boundary of mid-infrared optoelectronics our results demonstrate that cavity quantum electrodynamics can be used as tool in quantum material engineering, not only shifting and 
hybridising resonances, but non-perturbatively and controllably changing their nature.

Finally, one tantalising feature of the discrete resonances we observe is their conceptual proximity with Cooper pairs, bound states of two electrons bound by the exchange of virtual phonons [27]. Theoretical proposals have appeared, showing that the critical temperature of a superconductor could be theoretically modified by coupling electrons through the exchange of virtual polaritons [28] or virtual cavity photons $[25,29,30]$. And very recently, a first experimental result was reported [31]. The excitations we observed here differ in multiple fundamental aspects from Cooper pairs, including the fact they are electrically neutral and have a finite lifetime due to their polaritonic nature. Still, they remain a first demonstration of the possibility to bound two particles not via Coulomb interactions but through the exchange of photonic excitations mediated by a judiciously engineered resonator.
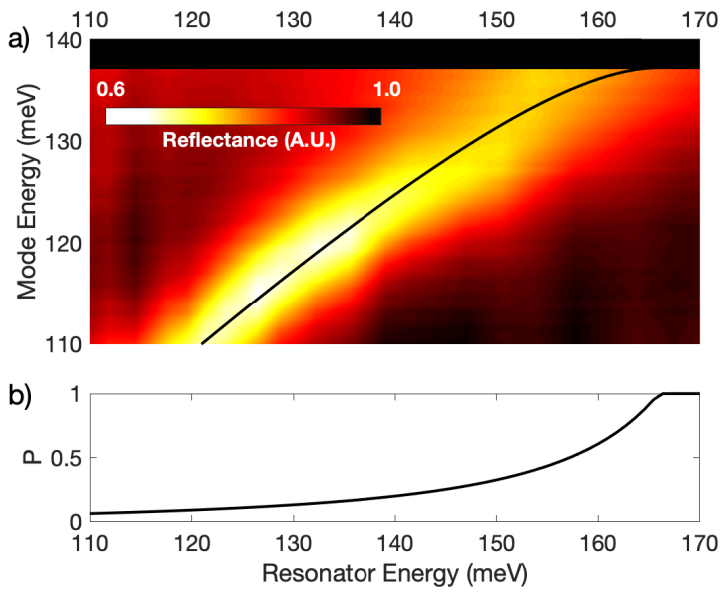

Figure 5. Calculation of the matter weight. In panel (a) we plot the eigenmodes obtained by our theoretical model with parameters choose to fit the experimental reflectance data shown in the colorplot. Above the ionization threshold the figure is uniformly black as the discrete modes used in the numerical diagonalization are too closely-spaced to be resolved. In panel (b) we use the parameters extracted from panel a) to calculate the matter fraction $P$ of the discrete polariton mode.

\section{ACKNOWLEDGEMENTS}

S.D.L. is a Royal Society Research Fellow and acknowledges partial financial support from the Philip Leverhulme Prize of the Leverhulme Trust; R.C., J.M.M., G.B. and I.C. acknowledge partial financial support from the European Union FET-Open Grant MIR-BOSE 737017; R.C. and A.B. acknowledge partial financial support from the French National Research Agency (project
"IRENA"). This work was partly supported by the French RENATECH network.

\section{METHODS}

\section{Sample growth and fabrication}

The samples were grown by molecular beam epitaxy (MBE) on semi-insulating 2-inch GaAs wafers. The growth starts with a 500-nm-thick $\mathrm{Al}_{0.50} \mathrm{Ga}_{0.50} \mathrm{As}$ stop layer, followed by 13 repetitions of GaAs QWs in $\mathrm{Al}_{0.33} \mathrm{Ga}_{0.67}$ As barriers, and it ends with a 30-nm-thick GaAs cap layer. The two samples differ in QW width and introduced electronic doping, while the barriers are always 10-nm-thick. Sample HM4229 contains 4-nmthick GaAs QWs $\left(L_{\mathrm{QW}}=4 \mathrm{~nm}\right)$, while sample HM4230 contains 3.5-nm-thick QWs $\left(L_{\mathrm{QW}}=3.5 \mathrm{~nm}\right)$. Si doping is introduced in the center of the AlGaAs barriers as $\delta$-doping, yielding a $2 \mathrm{D}$ electron density in the wells (measured at $300 \mathrm{~K}$ ) of $5 \times 10^{12} \mathrm{~cm}^{-2}$ (HM4229) and $4.77 \times 10^{12} \mathrm{~cm}^{-2}$ (HM4230), respectively.

The metal-semiconductor-metal resonators were realized by bonding the samples with Gold-gold thermocompression onto undoped GaAs wafers, following the procedure in Ref. [20]. After substrate removal, the resonators were defined with e-beam lithography followed by metal evaporation $(\mathrm{Ti} / \mathrm{Au})$ followed by lift-off.

\section{Ribbon resonators}

The use of metal-semiconductor-metal resonators is important as it permits to couple to intersubband transitions, that are TM polarized, even at normal incidence. In this case, however, the extreme thinness of the active region $(\approx 260 \mathrm{~nm})$ forbids operation in the photoniccrystal regime [32], and places the system in the independent resonator regime instead. The resonance frequency $f$ of the Fabry-Perot resonator is set by the width $p$, and not by the period $D$, according to the simple formula

$$
\frac{c}{f}=\frac{2 n_{e f f} p}{m}, \quad m \in \mathbb{N},
$$

with $c$ the speed of light and $n_{e f f}$ is an effective index that takes into account the reflectivity phase at the metallic boundaries.

We decided to employ the cavity mode with $m=2$, instead of the fundamental mode with $m=0$ that is typically employed in these systems, to simplify the fabrication procedure and increase the electromagnetic overlap factor. This is evident from Fig. 2(a) that highlights three nodal lines for the resonant cavity mode. 


\section{Spectroscopic characterization}

The transmission spectra of the samples before waferbonding are acquired with standard multi-pass waveguide transmission measurements, after the samples have been metallized with $\mathrm{Ti} / \mathrm{Au}$ on the top surface, and 45 degrees facets are shaped with mechanical polishing. The measurements are performed with an FTIR spectrometer, equipped with a thermal globar source, and a deuterated triglycine sulfate (DTGS) detector.

The reflectivity measurements have been performed with a microscope connected to the FTIR. The microscope optics/mirrors operate in the mid-IR, the source is a thermal globar, while the detector is a liquid-nitrogen cooled Mercury-Cadmium-Telluride (MCT). Light is focused on the sample with a Cassegrain objective that operates at a fixed incidence angle of incidence of $20^{\circ} \pm 5^{\circ}$. A very thin cryostat (Linkam LNP96) fits below the microscope objectives and permits to perform the reflectivity measurements at a temperature of $78 \mathrm{~K}$.

\section{Fitting procedure}

Both the resonances and the lifetimes showed in Fig. 4 have been obtained from the experimental reflectance data set for each metal stripe size, through an automatic multiple Lorentzian peak fitting procedure. Same fitting model has been used for both doped and undoped samples' data.

In order to calculate the matter component $P$ use used the same procedure developed in Ref. [4], using the doping and the ionization energy as adjustable parameters.

\section{DATA AVAILABILITY}

The data that support the findings of this study are available from the corresponding author upon reasonable request.

\section{CODE AVAILABILITY}

The codes that support the findings of this study are available from the corresponding author upon reasonable request.

* E-mail: raffaele.colombelli@u-psud.fr

† E-mail: s.de-liberato@soton.ac.uk

[1] Nikonov, D. E., Imamoğlu, A., Butov, L. V. \& Schmidt, $\mathrm{H}$. Collective intersubband excitations in quantum wells: Coulomb interaction versus subband dispersion. Phys. Rev. Lett. 79, 4633-4636 (1997).
[2] Frisk Kockum, A., Miranowicz, A., De Liberato, S., Savasta, S. \& Nori, F. Ultrastrong coupling between light and matter. Nat. Rev. Phys. 1, 19-40 (2019).

[3] Forn-Díaz, P., Lamata, L., Rico, E., Kono, J. \& Solano, E. Ultrastrong coupling regimes of light-matter interaction. Rev. Mod. Phys. 91, 025005 (2019).

[4] Cortese, E., Carusotto, I., Colombelli, R. \& De Liberato, S. Strong coupling of ionizing transitions. Optica 6, 354361 (2019).

[5] Capasso, F. et al. Observation of an electronic bound state above a potential well. Nature 358, 565-567 (1992).

[6] Ballarini, D. \& De Liberato, S. Polaritonics: from microcavities to sub-wavelength confinement. Nanophotonics 8, 641-654 (2019).

[7] Khurgin, J. Excitonic radius in the cavity polariton in the regime of very strong coupling. Solid state communications 117, 307-310 (2001).

[8] Citrin, D. S. \& Khurgin, J. B. Microcavity effect on the electron-hole relative motion in semiconductor quantum wells. Phys. Rev. B 68, 205325 (2003).

[9] Khurgin, J. B. Pliable polaritons: Wannier excitonplasmon coupling in metal-semiconductor structures. Nanophotonics 8, 629-639 (2018).

[10] Yang, M.-J., Kim, N. Y., Yamamoto, Y. \& Na, N. Verification of very strong coupling in a semiconductor optical microcavity. New J Phys 17, 023064 (2015).

[11] Brodbeck, S. et al. Experimental verification of the very strong coupling regime in a gaas quantum well microcavity. Phys. Rev. Lett. 119, 027401 (2017).

[12] Anappara, A. A. et al. Signatures of the ultrastrong lightmatter coupling regime. Phys. Rev. B 79, 201303 (2009).

[13] Todorov, Y. et al. Ultrastrong Light-Matter Coupling Regime with Polariton Dots. Phys. Rev. Lett. 105, 196402 (2010).

[14] Günter, G. et al. Sub-cycle switch-on of ultrastrong lightmatter interaction. Nature 458, 178-181 (2009).

[15] Levinsen, J., Li, G. \& Parish, M. M. Microscopic description of exciton-polaritons in microcavities. Phys. Rev. Research 1, 033120 (2019).

[16] Averkiev, N. S. \& Glazov, M. M. Light-matter interaction in doped microcavities. Phys. Rev. B 76, 045320 (2007).

[17] Haken, H. Quantum field theory of solids: An introduction (North-Holland, 1976).

[18] Todorov, Y. et al. Optical properties of metal-dielectricmetal microcavities in the thz frequency range. Opt. Express 18, 13886-13907 (2010).

[19] Dini, D., Köhler, R., Tredicucci, A., Biasiol, G. \& Sorba, L. Microcavity polariton splitting of intersubband transitions. Phys. Rev. Lett. 90, 116401 (2003).

[20] Manceau, J.-M. et al. Resonant intersubband polaritonlo phonon scattering in an optically pumped polaritonic device. Appl. Phys. Lett. 112, 191106 (2018).

[21] Hopfield, J. Theory of the contribution of excitons to the complex dielectric constant of crystals. Physical Review 112, 1555-1567 (1958).

[22] De Liberato, S. \& Ciuti, C. Quantum theory of electron tunneling into intersubband cavity polariton states. Phys. Rev. B 79, 075317 (2009).

[23] Vigneron, P.-B. et al. Quantum well infrared photodetectors operating in the strong light-matter coupling regime. Appl. Phys. Lett. 114, 131104 (2019).

[24] Galego, J., Garcia-Vidal, F. J. \& Feist, J. Cavityinduced modifications of molecular structure in the 
strong-coupling regime. Phys. Rev. X 5, 041022 (2015).

[25] Ebbesen, T. W. Hybrid light-matter states in a molecular and material science perspective. Accounts of Chemical Research 49, 2403-2412 (2016).

[26] Ruggenthaler, M., Tancogne-Dejean, N., Flick, J., Appel, H. \& Rubio, A. From a quantum-electrodynamical light-matter description to novel spectroscopies. Nat. Rev. Chem. 2, 1-16 (2018).

[27] Cooper, L. N. Bound electron pairs in a degenerate fermi gas. Phys. Rev. 104, 1189-1190 (1956).

[28] Laussy, F. P., Kavokin, A. V. \& Shelykh, I. A. Excitonpolariton mediated superconductivity. Phys. Rev. Lett. 104, 106402 (2010).

[29] Schlawin, F., Cavalleri, A. \& Jaksch, D. Cavity-mediated electron-photon superconductivity. Phys. Rev. Lett. 122, 133602 (2019).

[30] Curtis, J. B., Raines, Z. M., Allocca, A. A., Hafezi, M. \& Galitski, V. M. Cavity quantum eliashberg enhancement of superconductivity. Phys. Rev. Lett. 122, 167002 (2019).

[31] Thomas, A. et al. Exploring Superconductivity under Strong Coupling with the Vacuum Electromagnetic
Field. arXiv:1911.01459v1.

[32] Chassagneux, Y. et al. Electrically pumped photoniccrystal terahertz lasers controlled by boundary conditions. Nature 457, 174-178 (2009).

\section{AUTHOR CONTRIBUTIONS}

S.D.L. supervised the project and led the theoretical work. I.C., R.C., and S.D.L., designed the experiment. R.C. led the experimental work. G.B. grew the sample and N.L.T. fabricated the devices. N.L.T., J.M., and A.B. carried out the optical characterisation. E.C. performed the data analysis. All co-authors discussed the data and contributed to the manuscript.

\section{COMPETING INTERESTS}

The authors declare no competing interests. 